\title{
Cancer-adipose tissue interaction and fluid flow synergistically modulate cell kinetics, HER2 expression, and trastuzumab efficacy in gastric cancer
}

\author{
Takashi Akutagawa $^{1,2} \cdot$ Shigehisa Aoki $^{1} \cdot$ Mihoko Yamamoto-Rikitake $^{1} \cdot$ Ryuichi Iwakiri $^{2} \cdot$ Kazuma Fujimoto $^{2}$. \\ Shuji Toda ${ }^{1}$
}

Received: 12 March 2018 / Accepted: 20 April 2018 / Published online: 25 April 2018

(c) The International Gastric Cancer Association and The Japanese Gastric Cancer Association 2018

\begin{abstract}
Background Early local tumor invasion in gastric cancer results in likely encounters between cancer cells and submucosal and subserosal adipose tissue, but these interactions remain to be clarified. Microenvironmental mechanical forces, such as fluid flow, are known to modulate normal cell kinetics, but the effects of fluid flow on gastric cancer cells are poorly understood. We analyzed the cell kinetics and chemosensitivity in gastric cancer using a simple in vitro model that simultaneously replicated the cancer-adipocyte interaction and physical microenvironment.

Methods Gastric cancer cells (MKN7 and MKN74) were seeded on rat adipose tissue fragment-embedded discs or collagen discs alone. To generate fluid flow, samples were placed on a rotatory shaker in a $\mathrm{CO}_{2}$ incubator. Proliferation, apoptosis, invasion, and motility-related molecules were analyzed by morphometry and immunostaining. Proteins were evaluated by western blot analysis. Chemosensitivity was investigated by trastuzumab treatment.

Results Adipose tissue and fluid flow had a positive synergistic effect on the proliferative potential and invasive capacity of gastric cancer cells, and adipose tissue inhibited apoptosis in these cells. Adipose tissue upregulated ERK1/2 signaling in gastric cancer cells, but downregulated p38 signaling. Notably, adipose tissue and fluid flow promoted membranous and cytoplasmic HER2 expression and modulated chemosensitivity to trastuzumab in gastric cancer cells.

Conclusion We have demonstrated that cancer-adipocyte interaction and physical microenvironment mutually modulate gastric cancer cell kinetics. Further elucidation of the microenvironmental regulation in gastric cancer will be very important for the development of strategies involving molecular targeted therapy.
\end{abstract}

Keywords Cancer-associated adipocyte $\cdot$ Fluid flow $\cdot$ Human epidermal growth factor receptor $2($ HER2) $\cdot$ Mitogenactivated protein kinase (MAPK) · Trastuzumab

Takashi Akutagawa

14624001@edu.cc.saga-u.ac.jp

$\square$ Shigehisa Aoki

aokis@cc.saga-u.ac.jp

1 Department of Pathology and Microbiology, Faculty of Medicine, Saga University, 5-1-1 Nabeshima, Saga, Saga 849-8501, Japan

2 Department of Internal Medicine and Gastrointestinal Endoscopy, Faculty of Medicine, Saga University, 5-1-1 Nabeshima, Saga, Saga 849-8501, Japan

\section{Introduction}

The stomach is a hollow organ composed of mucosa, submucosa, muscular wall, and serosa (Fig. 1a). Gastric adipose tissue cells are special stromal cells that exist in the submucosa and subserosa (layer under the serosa). Recently, cancer-stromal cell interaction has attracted attention, because stromal cells, especially cancer-associated fibroblasts and adipocytes, can regulate growth, invasion, and metastasis in many cancer types $[1,2]$. Submucosal and subserosal adipose tissue is a frequent site of gastric cancer invasion (Fig. 1b). Although adipose tissue appears to have a critical role in gastric cancer progression, the underlying mechanism remains unclear. 
Fig. 1 Gastric wall and experimental design. a Histology and schematic illustration of the gastric wall. Adipose tissue is distributed in the submucosal layer. Streaming of gastric fluid (dashed arrow) and interstitial fluid (solid arrows) leads to fluid flow in the gastric mucosa. b Invasion of gastric cancer cells (arrowheads) into adipose tissue in vivo. c Coculture model and fluid flow-generating system. Gastric cancer cells are seeded on collagen gel discs embedded with adipose tissue fragments. As a control, cancer cells are seeded on collagen gel discs without adipose tissue fragments. To generate fluid flow, culture dishes are placed on a rotatory shaker in a $\mathrm{CO}_{2}$ incubator

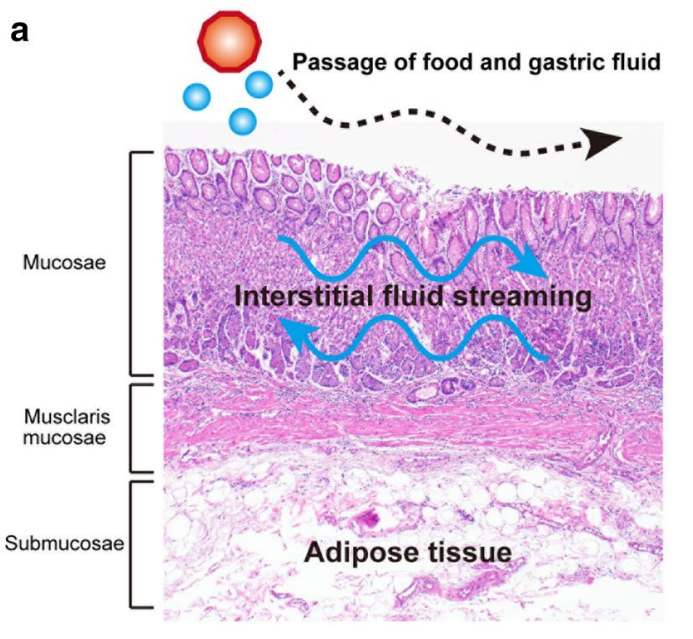

b

C

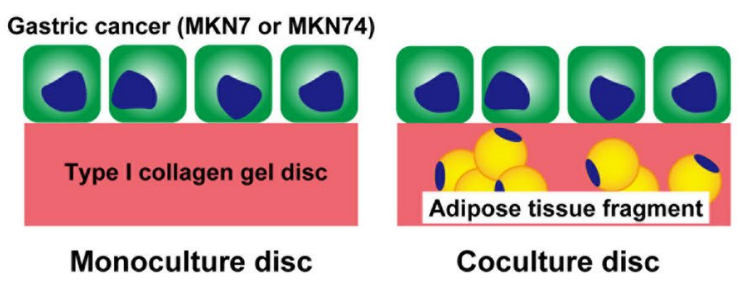

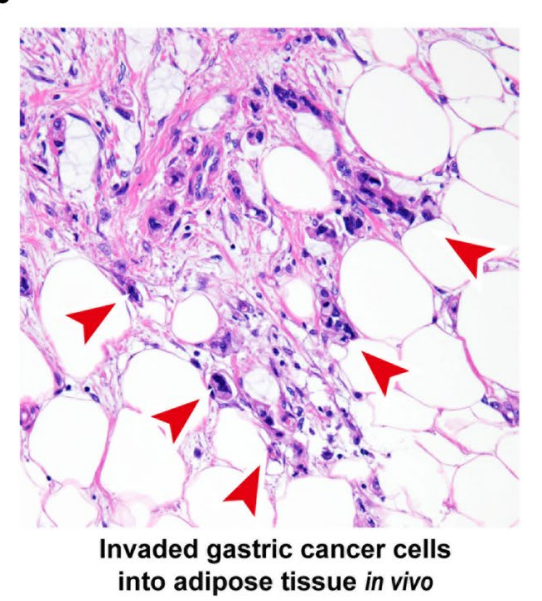

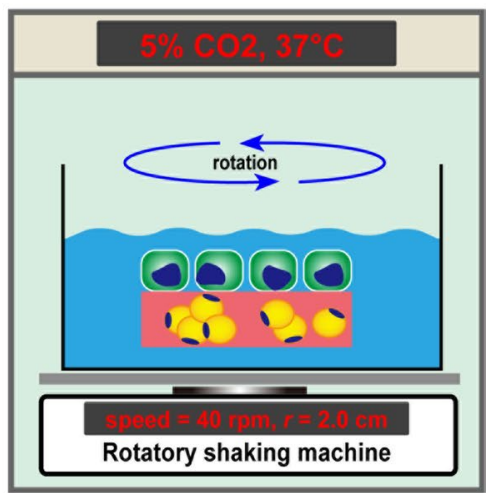

Physiological stress, especially fluid streaming, has been implicated in the physiologic responses of endothelial cells $[3,4]$. Shear stress and fluid streaming were recently demonstrated to constitute a critical microenvironment for various cell types, including stem cells [5, 6]. In the stomach, three types of epithelial cells (foveolar, parietal, and chief) secrete specific kinds of gastric fluid. Muscular wall contraction and relaxation create peristalsis of the stomach, and this movement induces gastric fluid streaming that physically breaks down ingested food. Interstitial fluid flow attributed to the vascular network is also normally present in the gastric mucosa [7].

We hypothesize that there may be a connection between cancer-stromal cell interaction and fluid flow and that these two factors may have an important role in the behavior of gastric cancer cells. In addition, trastuzumab, a monoclonal antibody against human epidermal growth factor receptor 2 (HER2), is often employed as a first-line combination chemotherapy drug for HER2-positive advanced gastric cancer [8]. Although HER2 expression in cancer cells is a key reference marker for trastuzumab efficacy, the influence of cancer-stromal cell interaction and fluid flow on HER 2 expression in cancer cells remains unclear. There have been no culture models that can simultaneously reconstruct cell-cell interaction and fluid streaming. To the best of our knowledge, we have overcome this issue by establishing a simple culture model that can concurrently replicate the cellular and physical microenvironment in gastric cancer.

The aim of this study was to clarify the impact of cancer-adipose tissue interaction and fluid flow on kinetics, HER2 expression, and trastuzumab efficacy in gastric cancer.

\section{Materials and methods}

\section{Cell lines and adipose tissue preparation}

All procedures involving human and animal materials were performed in accordance with the ethical guidelines of Saga University. Two human gastric cancer cell lines, MKN7 (well-differentiated type) and MKN74 (moderatelydifferentiated type), were obtained from the Japanese Cancer Research Bank (Osaka, Japan). Adipose tissue fragments were isolated from subcutaneous adipose tissue of 1-weekold Wistar rats. 


\section{Culture model}

To replicate the cell-cell interaction, we used a coculture system (Fig. 1c). First, adipose tissue was minced into pieces of about $0.5-\mathrm{mm}$ diameter. Next, $0.15 \mathrm{~g}$ of minced adipose tissue was mixed with $10.0 \mathrm{ml}$ of collagen gel solution (Cellmatrix, type I-A; Nitta Gelatin Co. Ltd., Osaka, Japan), and $1 \mathrm{ml}$ of the mixture was poured into $15-\mathrm{mm}$ diameter 24 -well plates. For cocultures, $2 \times 10^{5}$ gastric cancer cells were seeded onto the collagen gel disc surface. At 1 day after seeding, the collagen gel discs were transferred to $10-\mathrm{cm}$ diameter dishes in $40 \mathrm{ml}$ of complete medium comprising RPMI 1640 (Wako Pure Chemical Industries Ltd., Osaka, Japan) supplemented with $10 \%$ fetal bovine serum, $100 \mu \mathrm{g} /$ $\mathrm{ml}$ streptomycin, and $100 \mu \mathrm{g} / \mathrm{ml}$ penicillin. In control cultures, gastric cancer cells were seeded on collagen gel discs without adipose tissue fragments.

Species differences in humoral cross-reactivity need to be considered in this culture system. We addressed this issue in our previous studies and confirmed that rat-derived adipose tissue and adipocytes exhibited cross-reactivity with cells from other species, including human-derived and mousederived cells [9-11].

\section{Fluid flow-generating system}

The fluid flow-generating system was slightly modified from our previous method [11, 12]. The culture dishes were incubated under $5 \% \mathrm{CO}_{2}$ and $20 \% \mathrm{O}_{2}$ at $37{ }^{\circ} \mathrm{C}$ in a $\mathrm{CO}_{2}$ incubator. To generate fluid flow, the culture dishes were placed on a rotatory shaker (MIR-S100C; Panasonic, Tokyo, Japan) that rotated at a speed of $40 \mathrm{rpm}$ (Fig. 1c). Control dishes were placed in the $\mathrm{CO}_{2}$ incubator in the static condition. The culture medium was changed every 2 days.

\section{Histology and immunohistochemistry}

After 10 days of culture, histological examinations were performed by hematoxylin-eosin (HE) and Azan staining. Tissues were fixed with $10 \%$ formalin, routinely processed, and embedded in paraffin. HE and Azan staining procedures were performed on deparaffinized sections. Proliferative and apoptotic cells were respectively labeled with a mouse monoclonal anti-Ki-67 antibody (Dako, Glostrup, Denmark) and a rabbit monoclonal anti-cleaved caspase-3 antibody (Cell Signaling Technology, Danvers, MA). To distinguish spindle-shaped gastric cancer cells from mesenchymal cells, a mouse monoclonal anti-pan-cytokeratin (CK) AE1/AE3 antibody (Dako) was used. Expression of
HER2 in gastric cancer cells was examined using a rabbit monoclonal anti-HER2/erb2 antibody (Cell Signaling Technology). As negative controls, phosphate-buffered saline, normal mouse $\mathrm{IgG}$, and normal rabbit IgG were used as appropriate instead of a primary antibody.

\section{Morphometric analysis}

A total of 1,000 cells were counted (low magnification, $\times 10$ objective) in five randomly chosen non-contiguous and non-overlapping fields within the stained sections, and the percentages of Ki-67-positive cells and cleaved caspase 3-positive cells were determined for evaluation of proliferation and apoptosis, respectively. Cancer layer thicknesses were measured at 10 points in all five randomly chosen non-contiguous and non-overlapping areas (low magnification, $\times 10$ objective). Invasion depths of gastric cancer cells were measured by the distance from basement membrane to deepest front of gastric cancer cells. Numbers of invading gastric cancer cells were counted in $100-\mu \mathrm{m}$ squares.

\section{Western blot analysis}

To prepare proteins from cancer cells, adipose tissue fragments and cancer cells were cocultured using inserts with $8-\mu \mathrm{m}$ pore size (Falcon Cell Culture Insert; Becton Dickinson, Franklin, NJ). Adipose tissue fragments embedded in collagen gels were placed on the outside bottom of the inserts, and cancer cells were seeded inside the inserts. The inserts were placed in $10-\mathrm{cm}$ diameter dishes in $40 \mathrm{ml}$ of complete medium. After $48 \mathrm{~h}$ of culture, the collagen gels were stripped from the inserts. The cancer cells were lysed in $400 \mu \mathrm{l}$ of M-PER Reagent (Thermo Fisher Scientific, Waltham, MA) supplemented with a protease-phosphatase inhibitor cocktail (Cell Signaling Technology). Lysates containing an equal quantity of protein were separated by SDS-PAGE in $12 \%$ Bis-Tris gels and transferred to PVDF membranes. The membranes were incubated overnight at $4{ }^{\circ} \mathrm{C}$ with antibodies against HER2, extracellular-signalregulated kinase (ERK) 1/2, p-ERK1/2, p38, and p-p38 (Cell Signaling Technology). Antibody-bound antigens on the membranes were detected with a chemiluminescent immunodetection system (Western Breeze; Thermo Fisher Scientific). The band densities were determined with MultiImager II (BioTools Inc., Gunma, Japan) and Image J (http://rsb.info.nih.gov/ij/) software. The results were presented as ratios relative to control values. 


\section{Effect of HER2 inhibitor trastuzumab on gastric cancer cell behavior}

We examined the effects of the HER2 inhibitor trastuzumab (Herceptin; Chugai Pharmaceutical Co. Ltd., Tokyo, Japan) on gastric cancer cells. Trastuzumab was added to culture medium at a final concentration of $100.0 \mu \mathrm{g} / \mathrm{ml}$. Samples were collected and analyzed at day 5 .

\section{Statistical analysis}

Data from 3 to 5 independent experiments were analyzed by Tukey's test or Wilcoxon's test depending on equality of variance. Values are presented as mean \pm SEM, together with number of experiments carried out. Mean values of replicates in experiments were used to determine statistical significance, with values of $p<0.05$ considered to indicate statistically significant differences. All statistical analyses were performed using SPSS Statistics 23 software (IBM Corp., Armonk, NY).

\section{Results}

\section{Adipose tissue and fluid flow synergistically regulate cellular kinetics of gastric cancer cells}

To clarify stromal effects on gastric cancer, we cultured two types of gastric cancer cells with adipose tissue fragments. As shown in Fig. 2a, MKN7 cells cultured without adipose tissue had a flat cytoplasm and single-layer structure under static conditions. In contrast, MKN7 cells cultured with adipose tissue under the static condition showed increased cell density, cytoplasmic hypertrophy, and multi-stratified layer structure. Similarly, MKN74 cells cultured with adipose tissue under the static condition exhibited cytoplasmic hypertrophy, increased nuclear-cytoplasmic ratio, and thick stratified layer structure.

Fluid flow was added to the cell cultures to investigate the role of the dynamic condition. Under fluid flow, most MKN7 cells cultured with adipose tissue showed significantly increased cell density, cytoplasmic hypertrophy, and thickened cellular layer. Some MKN7 cells exhibited a papillary structure and invasion into the adipose tissue. Fluid flow induced increased cell density, increased nuclearcytoplasmic ratio, and multi-stratified layers in MKN74 cells cultured with or without adipose tissue. Small numbers of MKN74 cells cultured with adipose tissue showed invasion into the adipose tissue under fluid flow.

To examine cell growth and apoptosis of cancer cells, we performed immunostaining for Ki-67 and cleaved caspase-3. Coculture with adipose tissue significantly increased the Ki-67-positive cell number in MKN7 and MKN74 cells under the static condition (Fig. 2b). Fluid flow significantly increased the Ki-67-positive cell number in MKN7 and MKN74 cells, and adipose tissue and fluid flow had a positive synergistic effect on the proliferative potential in both cell types. As shown in Fig. 2c, adipose tissue inhibited cleaved caspase- 3 expression in MKN74 cells, and fluid flow did not affect the apoptotic ratio of MKN74 cells. Only a few MKN7 cells were positive for cleaved caspase- 3 with or without adipose tissue.

\section{Adipose tissue and fluid flow promote the invasive capacity of gastric cancer cells}

The invasive potential of gastric cancer has a central role in the poor prognosis for patients [13]. To investigate the invasive capacity, we measured invasion depths and numbers of cancer cells that invaded collagen gel discs (Fig. 3a). Intriguingly, MKN7 and MKN74 cells invaded collagen gel discs that contained adipose tissue, but did not invade collagen gel discs without adipose tissue (Fig. 2a). Fluid flow significantly increased both invasion depth and cell number of MKN7 and MKN74 cells relative to coculture with adipose tissue under the static condition (Fig. 3b-d).

\section{Adipose tissue and fluid flow modulate ERK1/2 and p38 expression in gastric cancer cells}

Mitogen-activated protein kinase (MAPK) pathways are involved in proliferation, epithelial-mesenchymal transition, and migration of gastric cancer cells [14]. To clarify the malignant potential of gastric cancer cell kinetics, we evaluated ERK1/2 and p38 expression in gastric cancer cells. As shown in Fig. 4a, b, adipose tissue promoted total expression and phosphorylation ratio of ERK1/2 in MKN7 and MKN74 cells under the static condition. Adipose tissue did not affect total p38 expression, but did inhibit phosphorylation ratio of p38 in both cancer cell types under the static condition. Fluid flow significantly promoted p38 phosphorylation ratio in MKN7 cells with adipose tissue, but did not affect p38 expression and phosphorylation ratio under other conditions. Fluid flow upregulated ERK1/2 phosphorylation ratio in MKN74 cells cocultured with adipose tissue, but did not affect ERK1/2 expression and phosphorylation ratio under other conditions.

\section{Adipose tissue and fluid flow change HER2 expression in gastric cancer cells}

Trastuzumab, a monoclonal antibody against HER2, was investigated for combination chemotherapy, because trastuzumab is the first-line treatment for HER2-positive advanced gastric cancer [8]. As shown in Fig. 5a, MKN7 cells cultured without adipose tissue showed membranous 
a

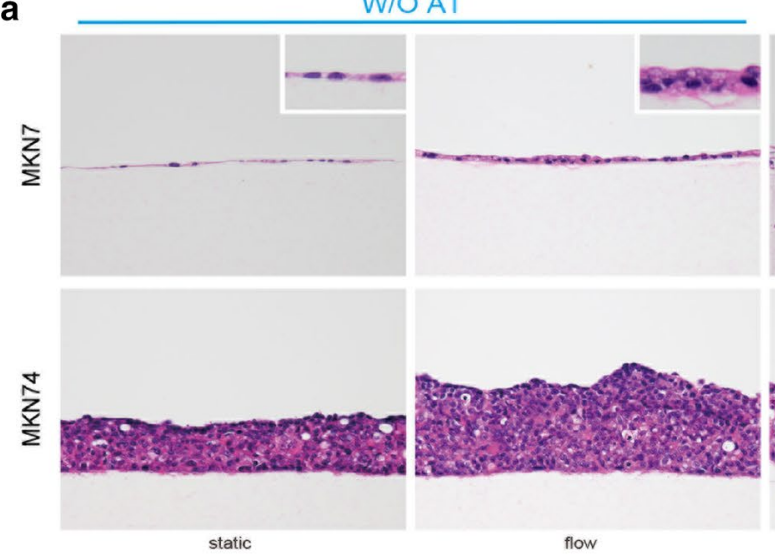

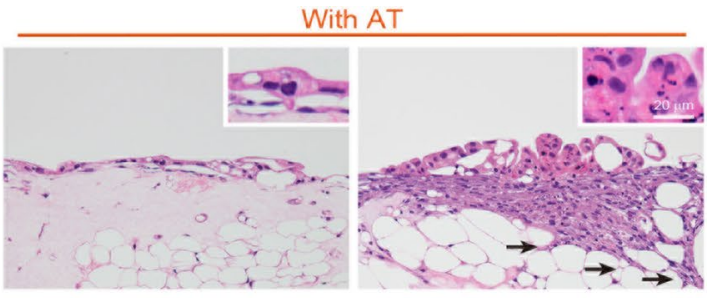
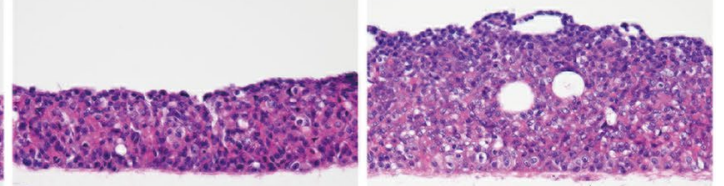

A $\quad 50 \mu \mathrm{m}$ static

flow

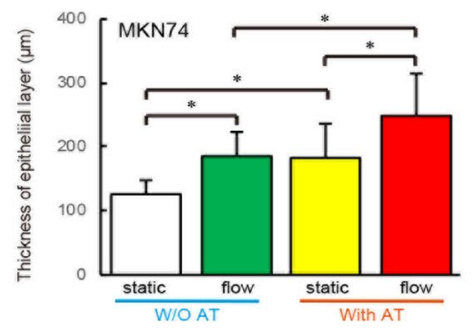

b
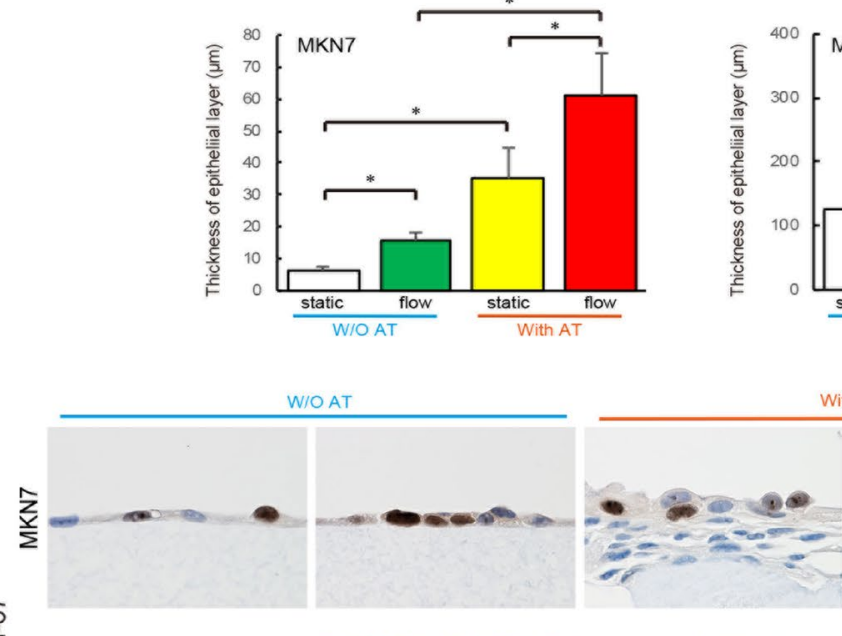

With AT
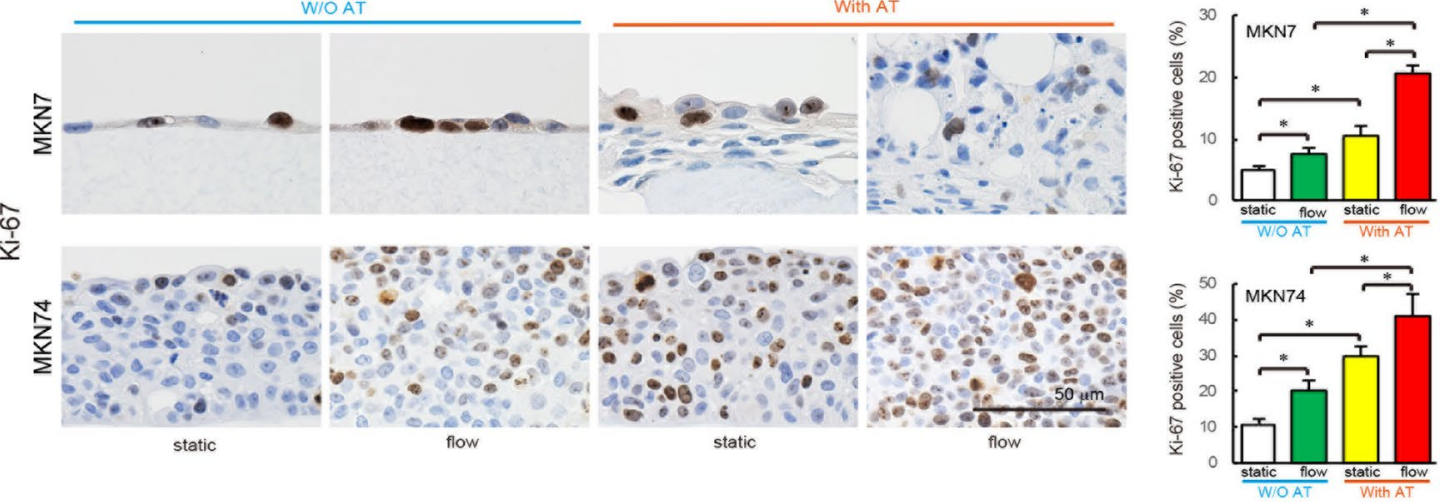

c
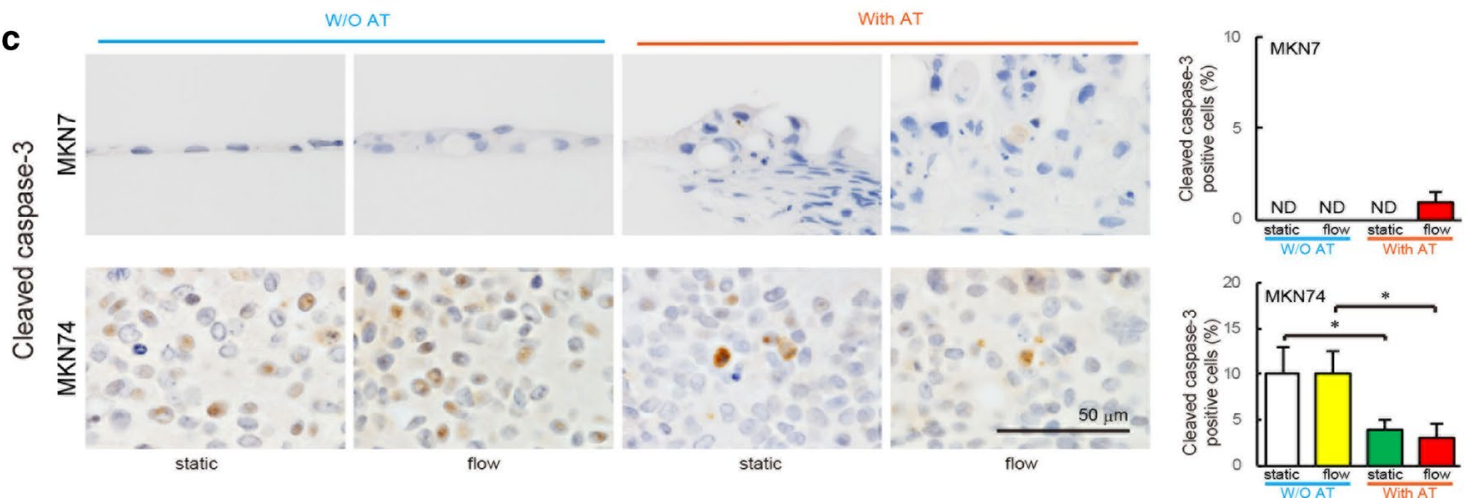

Fig. 2 Effect of adipose tissue and fluid flow on cellular kinetics of gastric cancer. a At day 10, adipose tissue and fluid flow synergistically promote cellular hypertrophy and thickened epithelium layer in MKN7 and MKN74 cells. MKN7 cells (arrows) and MKN74 cells (arrowhead) invade the adipose tissue. Insets: High magnification

images of MKN7 cells. b Immunostaining for $\mathrm{Ki}-67$ and percentages of Ki-67-positive cells. $\mathbf{c}$ Immunostaining for cleaved caspase-3 and percentages of cleaved caspase-3-positive cells. Data represent means \pm SEM of three determinations. $* p<0.05$ 
Fig. 3 Effect of adipose tissue and fluid flow on invasive capacity of gastric cancer cells. a Representative image for measuring the invasion depth and counting the number of invading cells. b Immunostaining for CK AE1/AE3 shows invasion of MKN7 and MKN74 cells into collagen gel discs. c Invasion depths and numbers of invading cells. Data represent means $\pm \mathrm{SD}$ of three determinations. $* p<0.05$. Scale bar $=50 \mu \mathrm{m}$ a
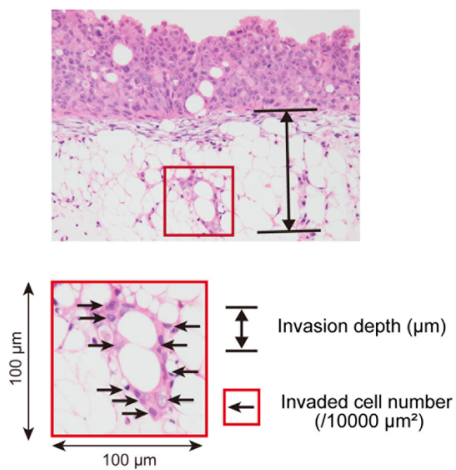

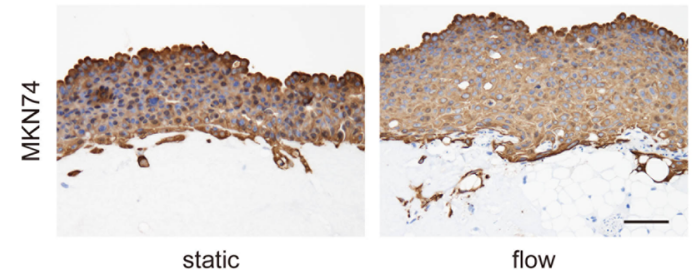

static

With AT

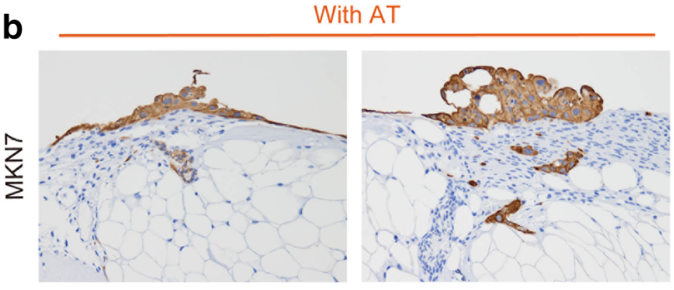

d
C

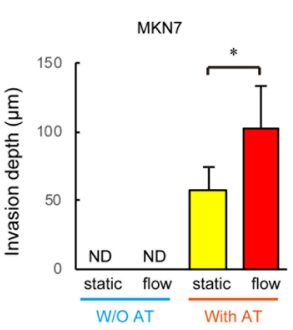

MKN74

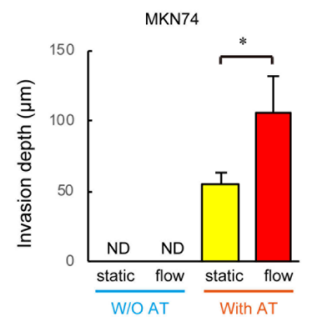

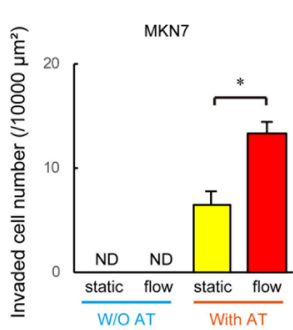

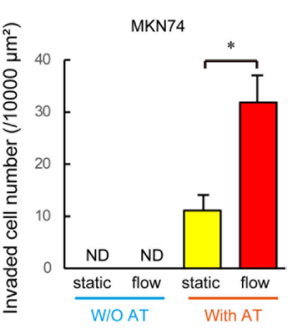

expression of HER2. Notably, adipose tissue and fluid flow induced HER2 cytoplasmic expression in addition to membranous expression in MKN7 cells, and membranous and cytoplasmic HER2 expressions were synergistically enhanced by the combination of adipose tissue and fluid flow. MKN74 cells cultured without adipose tissue showed faint HER2 expression under the static condition. Fluid flow upregulated membrane HER2 expression in MKN74 cells, while adipose tissue had no effect on HER2 expression in these cells.

To evaluate the chemosensitivity of MKN7 and MKN74 cells to trastuzumab, we administered trastuzumab under the fluid flow condition, based on our finding that fluid flow upregulated HER2 expression in gastric cancer cells. As shown in Fig. 5b, MKN7 cells cultured without adipose tissue showed thinning of the cytoplasm under trastuzumab administration, whereas MKN7 cells cultured with adipose tissue showed cellular hypertrophy and significantly increased number of Ki-67-positive cells. MKN74 cells cultured without adipose tissue exhibited no changes in morphology or Ki-67-positive cell ratio regardless of trastuzumab administration, whereas MKN74 cells cultured with adipose tissue showed thinner cellular layers and significantly decreased number of Ki-67-positive cells (Fig. 5c). Trastuzumab administration increased the number of cleaved caspase-3-positive cells in MKN7 cells cultured with adipose tissue. No significant difference was observed in the number of cleaved caspase-3-positive cells in MKN74 cells cultured with or without adipose tissue (Fig. 5d).

\section{Discussion}

In the present study, we have demonstrated for the first time that adipose tissue and fluid flow influence the behavior of gastric cancer cells under normal and chemotherapeutic conditions. Notably, adipose tissue and fluid flow had synergistic or independent effects on gastric cancer cells depending on the cell type.

Many studies have focused on the paracrine effect of adipocytes in various cancer types $[15,16]$. Recently, adipocytes located close to invasive cancer cells have been designated cancer-associated adipocytes, and these special adipocytes are recognized as an important factor for tumor development and progression [17-19]. However, no threedimensional models involving both cancer cells and adipocytes have been established to facilitate evaluation of the growth, invasion, and apoptosis of these cells under coexistence. Our simple culture model accurately evaluated the proliferation and apoptotic ratio of gastric cancer cells and clearly demonstrated cancer cell invasion into adipose tissue in the same manner as that observed in vivo.

Bowel movement is considered to be closely related to the risk of colorectal cancer [20]. However, despite several theories for an association [21-24], it is extremely difficult to identify a particular effect of bowel movement that may affect the risk of gastric cancer. Simons et al. [25] reported that frequent bowel movements were associated with an increased risk of rectal cancer, while constipation was associated with a decreased risk of rectal cancer. Bowel 
Fig. 4 Effect of adipose tissue and fluid flow on MAPK expression in gastric cancer cells. (a and b) Protein expression levels evaluated by western blot analysis in MKN7 (a) and MKN74 (b) cells. Relative expression is depicted as the ratio of target protein expression to $\beta$-actin expression. Data represent means \pm SD of 3-5 determinations. $* p<0.05$

\section{a}

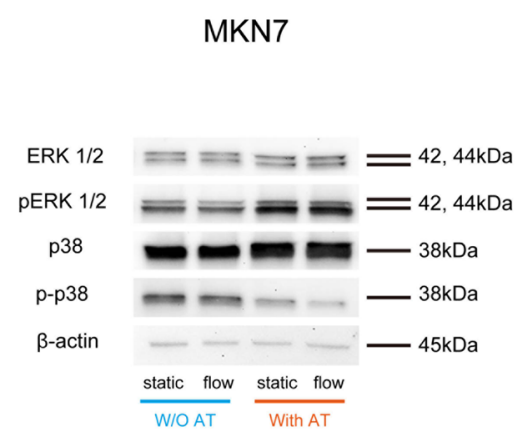

b

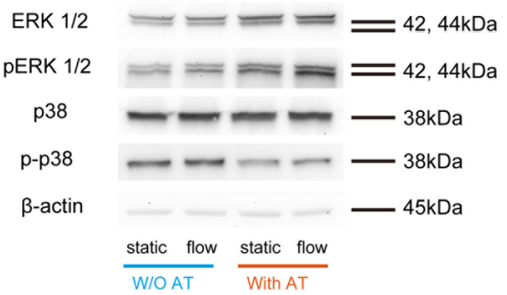

ERK 1/2

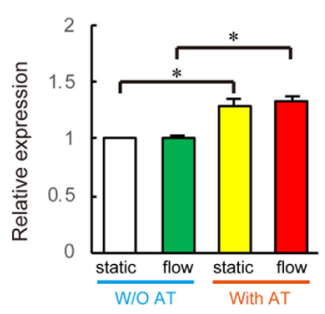

p38

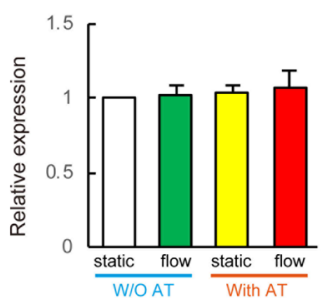

ERK $1 / 2$

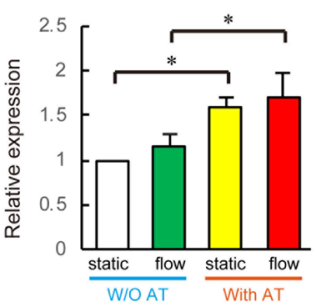

p38

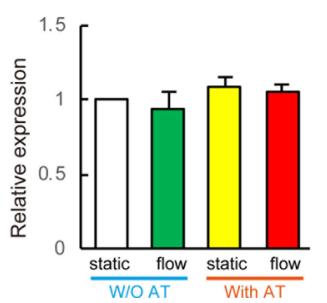

PERK $1 / 2$ / ERK $1 / 2$

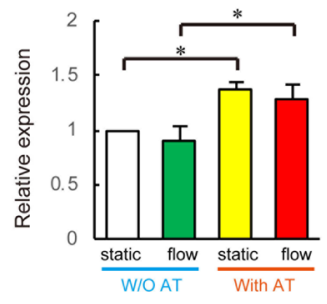

p-p38 / p38

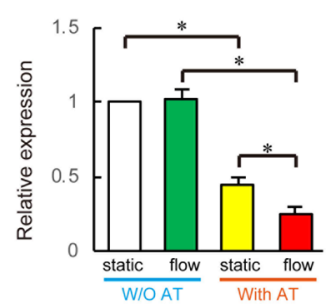

pERK1/2 / ERK1/2

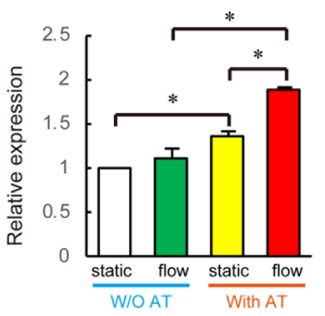

p-p38 / p38

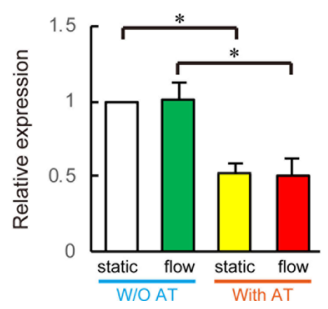

movement produces fluid flow in the digestive organs with stirring of the digestive juice and interstitial fluid. These findings suggest that there may be a close relationship between fluid flow and cancer development in the digestive organs. Fluid streaming and shear stress were recently demonstrated to constitute a critical microenvironment for various normal cell types [7, 26]. However, the physical effects on gastric cancer have received scant attention. Although interstitial fluid streaming in a given organ is an important factor for not only normal cells, but also cancer cells [27, 28], there have been no manageable three-dimensional culture models that can reconstruct cell-cell interaction and physical stress. In our simple culture model, small collagen gel discs can be easily produced and used to mimic gastric cancer organs under fluid flow.

As shown in Fig. 6, MAPK signaling and HER2 expression are considered crucial factors for cancer cell behavior at the molecular level [29]. HER2 is a member of the human epithelial growth factor family, and its overexpression is associated with poor prognosis in many cancer types including gastric cancer [30, 31]. In a previous report, adipose tissue and adipose tissue stromal cells downregulated HER2 expression in cancers other than gastric cancer [10]. However, in the present study, adipose tissue was found to increase cytoplasmic expression of HER2 in gastric cancer cells. An impact of cytoplasmic HER2 overexpression was reported in pancreatic and colorectal cancers [32, 33], but the underlying mechanism and detailed role of cytoplasmic HER2 have remained unclear. Our findings suggest that adipose tissue may lead to an increase in cytoplasmic HER2, which in turn enhances cell growth and proliferation. In this regard, it was notable that fluid flow acted as an independent factor for upregulated HER2 expression. Our findings suggested that adipose tissue and fluid flow were 
Fig. 5 Effect of adipose tissue and fluid flow on HER2 and chemosensitivity to trastuzumab in gastric cancer cells. a Immunostaining for HER2 in MKN7 and MKN74 cells. b Alterations of morphology, and $\mathrm{Ki}$-67-positive and cleaved caspase-3-positive cell ratios in MKN7 and MKN74 cells under trastuzumab administration at day 5. c Percentages of Ki-67-positive cells among MKN7 and MKN74 cells. (d) Percentages of cleaved caspase-3-positive cells among MKN7 and MKN74 cells. Data represent means $\pm \mathrm{SD}$ of three determinations. $* p<0.05$

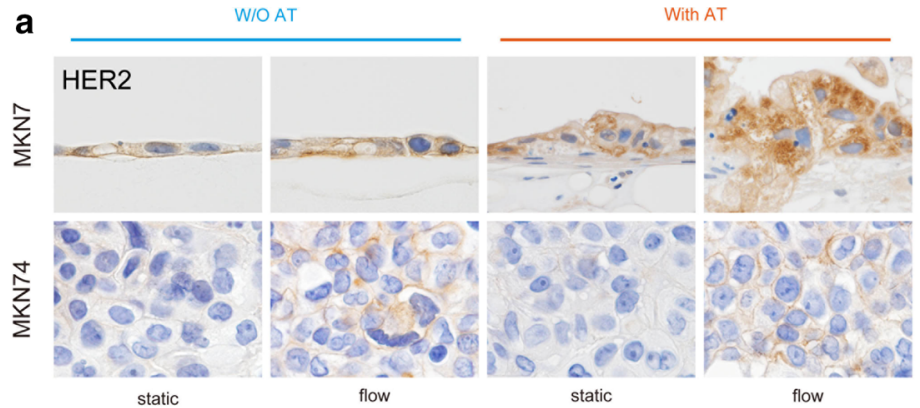

b
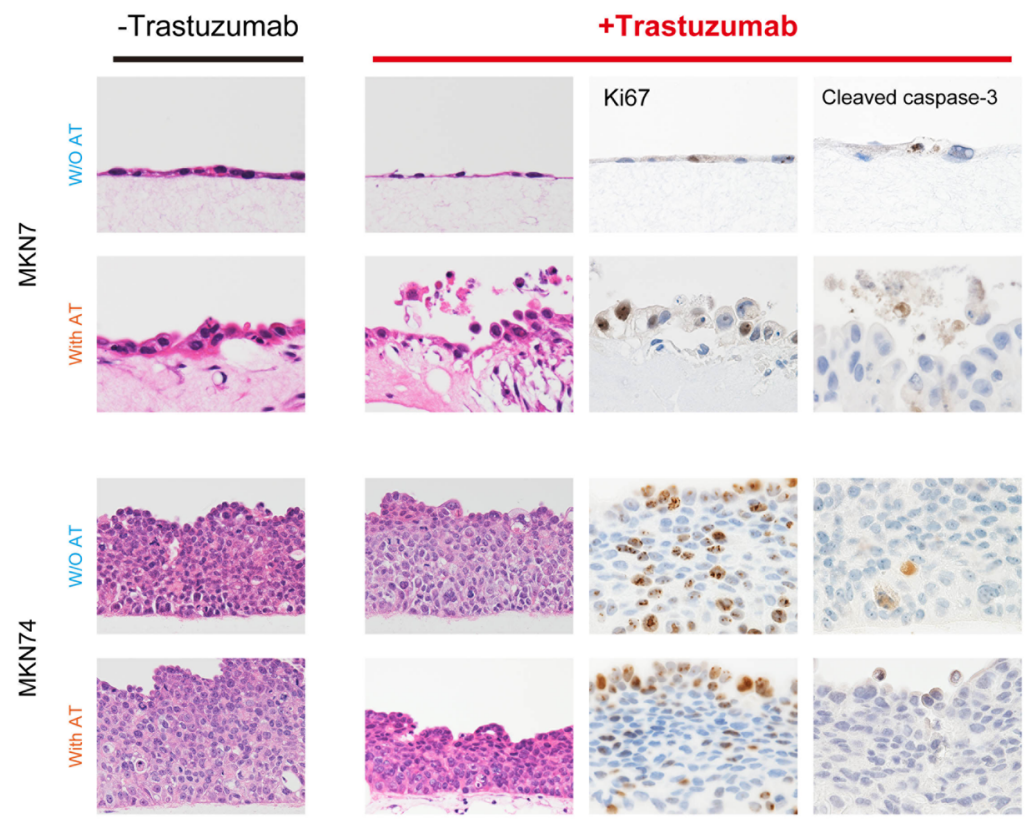

d

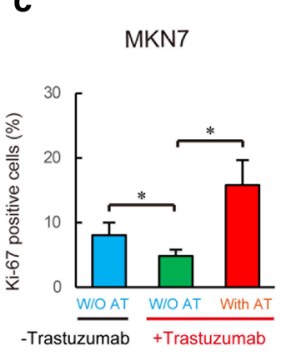

MKN74

MKN7

MKN74
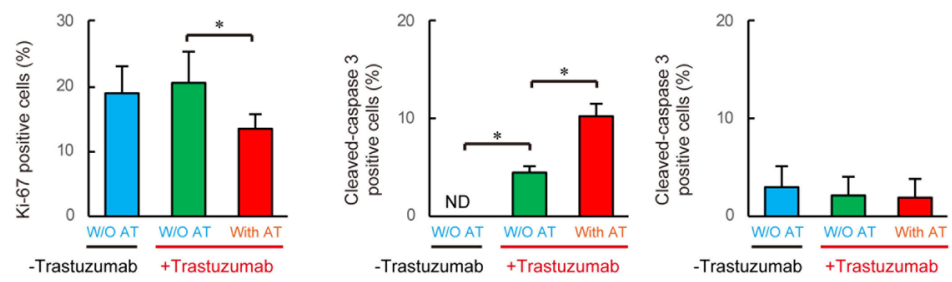

associated with HER2 expression in gastric cancer cells. Increased HER2 expression may be a factor in sensitivity to trastuzumab treatment. Cytoplasmic HER2 overexpression in gastric cancer is observed in clinicopathologic examinations [34, 35], but the guideline for the scoring system does not mention this possibility [36]. Our results suggest that cytoplasmic expression of HER2 may be a potential target for trastuzumab.

A previous report showed that adipose cells promoted resistance to trastuzumab-mediated cytotoxicity in breast cancer cells in vitro $[37,38]$. However, the chemo-mechanical crosstalk involving adipose tissue in gastric cancer remains less well understood. In the present study, adipose tissue sensitized MKN7 cells to apoptosis induced by trastuzumab. In contrast, adipose tissue reduced the number of Ki-67-positive MKN74 cells, resulting in suppression of the proliferation induced by trastuzumab. There seemed to be a definite discrepancy between these cancer cell phenotypes. Our findings suggest that adipose tissue may be associated with the prognosis in trastuzumab treatment for gastric cancer.

In conclusion, we have established a concurrent reconstruction model for the cellular and physiological microenvironment. Evaluation of trastuzumab administration in this 


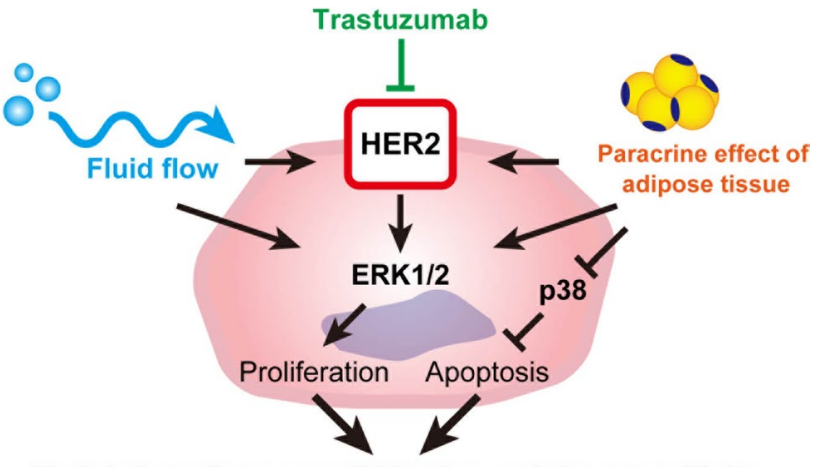

Modulation of cancer cell kinetics and drug sensitivity

Fig. 6 Summary of microenvironmental effects on gastric cancer. Paracrine effect of adipose tissue and physical stress of fluid flow modulate proliferative activity of gastric cancer cells through ERK1/2 signaling. Adipose tissue downregulates phosphorylation of p38 and may inhibit apoptosis of gastric cancer cells. Adipose tissue and fluid flow upregulate membranous or cytoplasmic HER2 expression and modulate chemosensitivity to trastuzumab

model demonstrated for the first time that adipose tissue and fluid flow have individual roles in drug sensitivity related to cancer survival. This alternative cancer model could become a promising tool for further novel drug discovery against many types of cancers.

Acknowledgements We thank T. Sakumoto, S. Morito, M. Nishida, F. Mutoh, S. Nakahara, and I. Nanbu for excellent technical assistance. We are grateful to Mr. K. Tokaichi for refining the English of the manuscript. We also thank Alison Sherwin, PhD, from Edanz Group (http:// www.edanzediting.com/ac) for editing a draft of this manuscript.

Funding This work was supported in part by the Center for Clinical and Translational Research of Kyushu University Hospital (to S.A.), and Grants-in-Aid from the Japanese Ministry of Education, Culture, Sports, Science and Technology for Scientific Research (no. 17K09352 to S.T. and no. $16 \mathrm{~K} 09284$ to S.A.).

\section{Compliance with ethical standards}

Conflict of interest The authors declare that they have no conflict of interest.

Ethical standards All institutional and national guidelines for the care and use of laboratory animals were followed in this study.

\section{References}

1. Östman A, Augsten M. Cancer-associated fibroblasts and tumor growth-bystanders turning into key players. Curr Opin Genet Dev. 2009;19(1):67-73.

2. Nieman KM, Romero IL, Van Houten B, Lengyel E. Adipose tissue and adipocytes support tumorigenesis and metastasis. Biochim Biophys Acta Mol Cell Biol Lipids. 2013;1831(10):1533-41.

3. Chatzizisis YS, Coskun AU, Jonas M, Edelman ER, Feldman $\mathrm{CL}$, Stone PH. Role of endothelial shear stress in the natural history of coronary atherosclerosis and vascular remodeling: molecular, cellular, and vascular behavior. J Am Coll Cardiol. 2007;49(25):2379-93.

4. Dewey C, Bussolari S, Gimbrone M, Davies PF. The dynamic response of vascular endothelial cells to fluid shear stress. J Biomech Eng. 1981;103(3):177-85.

5. Yamamoto K, Sokabe T, Watabe T, Miyazono K, Yamashita JK, Obi S, et al. Fluid shear stress induces differentiation of Flk-1-positive embryonic stem cells into vascular endothelial cells in vitro. Am J Physiol Heart Circ Physiol. 2005;288(4):H1915-24.

6. Knippenberg M, Helder MN, Zandieh Doulabi B, Semeins CM, Wuisman PI, Klein-Nulend J. Adipose tissue-derived mesenchymal stem cells acquire bone cell-like responsiveness to fluid shear stress on osteogenic stimulation. Tissue Eng. 2005;11(11-12):1780-88.

7. Kumar V, Abbas AK, Aster JC. Robbins basic pathology e-book. New York: Elsevier; 2017.

8. Bang Y-J, Van Cutsem E, Feyereislova A, Chung HC, Shen L, Sawaki A, et al. Trastuzumab in combination with chemotherapy versus chemotherapy alone for treatment of HER2-positive advanced gastric or gastro-oesophageal junction cancer (ToGA): a phase 3, open-label, randomised controlled trial. Lancet. 2010;376(9742):687-97.

9. Nomoto-Kojima N, Aoki S, Uchihashi K, Matsunobu A, Koike $\mathrm{E}$, Ootani A, et al. Interaction between adipose tissue stromal cells and gastric cancer cells in vitro. Cell Tissue Res. 2011;344(2):287-98.

10. Nakayama A, Aoki S, Uchihashi K, Nishijima-Matsunobu A, Yamamoto M, Kakihara N, et al. Interaction between esophageal squamous cell carcinoma and adipose tissue in vitro. Am J Pathol. 2016;186(5):1180-94.

11. Aoki S, Udo K, Morimoto H, Ikeda S, Takezawa T, Uchihashi K, et al. Adipose tissue behavior is distinctly regulated by neighboring cells and fluid flow stress: a possible role of adipose tissue in peritoneal fibrosis. J Artif Organs. 2013;16(3):322-31.

12. Aoki S, Makino J, Nagashima A, Takezawa T, Nomoto N, Uchihashi K, et al. Fluid flow stress affects peritoneal cell kinetics: possible pathogenesis of peritoneal fibrosis. Perit Dial Int. 2011;31(4):466-76.

13. Gotoda T, Yanagisawa A, Sasako M, Ono H, Nakanishi Y, Shimoda $\mathrm{T}$, et al. Incidence of lymph node metastasis from early gastric cancer: estimation with a large number of cases at two large centers. Gastric Cancer. 2000;3(4):219-25.

14. Zhang W, Liu HT. MAPK signal pathways in the regulation of cell proliferation in mammalian cells. Cell Res. 2002;12(1):9.

15. Kelesidis I, Kelesidis T, Mantzoros C. Adiponectin and cancer: a systematic review. Br J Cancer. 2006;94(9):1221.

16. Housa D, Housova J, Vernerova Z, Haluzik M. Adipocytokines and cancer. Physiol Res. 2006;55(3):233.

17. Tan J, Buache E, Chenard M-P, Dali-Youcef N, Rio M-C. Adipocyte is a non-trivial, dynamic partner of breast cancer cells. Int $\mathrm{J}$ Dev Biol. 2011;55(7-8-9):851-59.

18. Dirat B, Bochet L, Dabek M, Daviaud D, Dauvillier S, Majed B, et al. Cancer-associated adipocytes exhibit an activated phenotype and contribute to breast cancer invasion. Cancer Res. 2011;71(7):2455-65.

19. Cozzo AJ, Fuller AM, Makowski L. Contribution of adipose tissue to development of cancer. Compr Physiol. 2017;8(1):237-82.

20. Burkitt DP. Epidemiology of cancer of the colon and rectum. Cancer. 1971;28(1):3-13.

21. Kojima M, Wakai K, Tokudome S, Tamakoshi K, Toyoshima H, Watanabe Y, et al. Bowel movement frequency and risk of colorectal cancer in a large cohort study of Japanese men and women. Br J Cancer. 2004;90(7):1397.

22. Nascimbeni R, Donato F, Ghirardi M, Mariani P, Villanacci V, Salerni B. Constipation, anthranoid laxatives, melanosis coli, and 
colon cancer: a risk assessment using aberrant crypt foci. Cancer Epidemiol Prev Biomark. 2002;11(8):753-7.

23. Giovannucci E, Ascherio A, Rimm EB, Colditz GA, Stampfer MJ, Willett WC. Physical activity, obesity, and risk for colon cancer and adenoma in men. Ann Intern Med. 1995;122(5):327-34.

24. Anderson JC, Lacy BE. Editorial: Constipation and colorectal cancer risk: a continuing conundrum. Am J Gastroenterol. 2014;109(10):1650-2.

25. Simons CC, Schouten LJ, Weijenberg MP, Goldbohm RA, van den Brandt PA. Bowel movement and constipation frequencies and the risk of colorectal cancer among men in the Netherlands Cohort Study on Diet and Cancer. Am J Epidemiol. 2010;172(12):1404-14.

26. Fan R, Emery T, Zhang Y, Xia Y, Sun J, Wan J. Circulatory shear flow alters the viability and proliferation of circulating colon cancer cells. Sci Rep. 2016;6:27073.

27. Ross MH, Pawlina W. Histology. Philadelphia: Lippincott Williams \& Wilkins; 2006.

28. Polacheck WJ, Zervantonakis IK, Kamm RD. Tumor cell migration in complex microenvironments. Cell Mol Life Sci. 2013;70(8):1335-56.

29. Candas D, Lu C-L, Fan M, Chuang FY, Sweeney C, Borowsky AD, et al. Mitochondrial MKP1 is a target for therapy-resistant HER2positive breast cancer cells. Cancer Res. 2014;74(24):7498-509.

30. Seliger B, Kiessling R. The two sides of HER2/neu: immune escape versus surveillance. Trends Mol Med. 2013;19(11):677-84.

31. Bilous M, Dowsett M, Hanna W, Isola J, Lebeau A, Moreno A, et al. Current perspectives on HER2 testing: a review of national testing guidelines. Mod Pathol. 2003;16(2):173.
32. Blok EJ, Kuppen PJ, Van Leeuwen JE, Sier CF. Cytoplasmic overexpression of HER2: a key factor in colorectal cancer. Clin Med Insights Oncol. 2013;7:CMO. S10811.

33. Ueda S, Ogata S, Tsuda H, Kawarabayashi N, Kimura M, Sugiura Y, et al. The correlation between cytoplasmic overexpression of epidermal growth factor receptor and tumor aggressiveness: poor prognosis in patients with pancreatic ductal adenocarcinoma. Pancreas. 2004;29(1):e1-e8.

34. Half E, Broaddus R, Danenberg KD, Danenberg PV, Ayers GD, Sinicrope FA. HER-2 receptor expression, localization, and activation in colorectal cancer cell lines and human tumors. Int $\mathrm{J}$ Cancer. 2004;108(4):540-8.

35. Jindal Y, Varma K, Misra V, Kumar R, Singh A, Misra SP, et al. Cytoplasmic expression of HER2 in gastric adenocarcinoma: an unusual finding. IJMRPS. 2016;3(8):67-77.

36. Rüschoff J, Dietel M, Baretton G, Arbogast S, Walch A, Monges $\mathrm{G}$, et al. HER2 diagnostics in gastric cancer-guideline validation and development of standardized immunohistochemical testing. Virchows Arch. 2010;457(3):299-307.

37. Duong MN, Cleret A, Matera E-L, Chettab K, Mathé D, Valsesia-Wittmann S, et al. Adipose cells promote resistance of breast cancer cells to trastuzumab-mediated antibody-dependent cellular cytotoxicity. Breast Cancer Res. 2015;17(1):57.

38. Majidinia M, Yousefi B. Breast tumor stroma: a driving force in the development of resistance to therapies. Chem Biol Drug Des. 2017;89(3):309-18. 